\title{
Accretion Power in GRBs
}

\author{
Tsvi Piran \\ Racah Institute for Physics, The Hebrew University, Jerusalem, 91904, Israel \\ and Theoretical Astrophysics, Caltech, Pasadena, CA 91125, USA
}

\begin{abstract}
I discuss the implication of the temporal structure of GRBs to the nature of their inner engine. I argue that the temporal strucutre shows that GRBs must involve internal shocks (or another kind of internal interaction within a relativistic outflow). To produce these internal shocks GRB inner engines must vary on a time scale of a fraction of a second and, on the other hand, they should be active for the whole duration of the burst, namely for several dozen of seconds. This implies that from the point of view of the central engine GRBs are a "quasi steady state" phenomenon. Accretion onto a newly formed black hole is the most likely mechanism that can satisfy these conditions and can power GRBs. I discuss the implication of accretion models of massive disks around black holes to GRB modelling.
\end{abstract}

Keywords: Gamma-ray bursts, accretion

PACS: $97.10 . \mathrm{Gz}, 98.70 . \mathrm{Rz}$

\section{INTRODUCTION}

Our understanding of Gamma-Ray Bursts (GRBs) was revolutionized during the last ten years. According to the generally accepted Fireball model (see e.g. [1, 2] for recent reviews) the gamma-rays and their subsequent multiwavelength afterglow are produced when the kinetic energy of ultra-relativistic flow is dissipated. Most current observations, from prompt emission to late afterglow, from $\gamma$-rays via X-ray optical and IR to radio, are consistent with this model.

Today there is a clear evidence that (at least some) long duration GRBs are associated with Supernovae $[3,4,5,6,7,8]$. This has been suggested by the Collapsar model $[9,10,11]$ and confirmed by the association of long duration GRBs with type Ic supernovae $[7,8]$. The origin of short GRBs is not clear but merging binary neutron stars [12] are possible candidates. In both cases it is believed that the GRB's "inner engine" involve accretion onto a newborn black hole, even though the details of the acceleration and collimation of the relativistic outflow are not understood.

I begin with summarizing the constrains on the "inner engines" of GRBs that arise from the temporal structure of GRBs. Sari and Piran [13] have shown that variable GRBs can be generated only by internal interactions ${ }^{1}$ within the flow. To produce internal shocks the central engine must produce a long and variable wind. Kobayashi et al [14] have shown that the observed internal shocks light curve reflects almost directly the temporal activity of the inner engine. This is the best direct evidence on what is

\footnotetext{
${ }^{1}$ This interaction is usually considered as a collisionless shock. However the exact nature of the interaction is unimportant for most of our arguments.
} 
happening at the center of the GRB. I review the arguments leading to these conclusions.

I then discuss additional observational results [15, 16, 17] and a theoretical toy model [18] that explains these observations within the internal shocks paradigm. The implication of these results is that the inner engine must be active for a very long time scale (the duration of the burst) compared to its own dynamical time scale (the dynamical scale of a typical compact object - black hole or a neutron star - is less than a millisecond). The most natural engine that can satisfy these conditions is a rather massive compact accretion disk around a newly formed compact object. In this case the observed time of the burst is the accretion time of the disk, while the fluctuation time is the dynamical time scale of the disk. The energy requirement suggests that the disk contains about $0.1 M_{\odot}$ and such a massive disk can arise only during the formation process of the compact object.

I conclude with a discussion of the implications of accretion theory to the black holeaccretion disk model for the inner engine of GRBs. I show that this theory implies that long GRBs should be produced by a Collapsar while short one by merging neutron star binaries. I refer the reader to [2] for a recent detailed review on other properties of GRBs.

\section{TIME SCALES IN GRBS - OBSERVATIONS}

Most GRBs are highly variable. Fig. 1 depicts the light curve of a typical variable GRB $(\text { GRB920627 })^{2}$. The variability time scale, $\delta t$, as determined by the width of the peaks is much shorter (in some cases by a more than a factor of 100) then $T$, the duration of the burst. Variability on a time scale of milliseconds is seen in some long bursts [17].

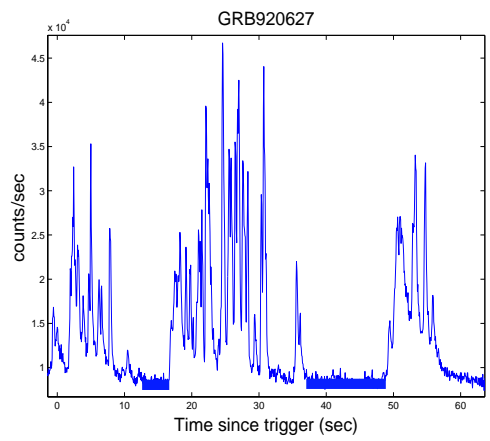

FIGURE 1. The light curve of GRB920627. The total duration of the burst is 52sec, while typical pulses are $0.8 \mathrm{sec}$ wide. Two quiescent periods lasting $\sim 10$ seconds are marked by horizontal solid bold lines.

A comparison of the pulse width distribution and the pulse separation, $\Delta t$, distribution, reveals an excess of long intervals $[15,16]$. These long intervals can be classified as quiescent periods [19], relatively long periods of several dozen seconds with no activity. When excluding the quiescent periods Nakar and Piran [15, 16] find that both distributions are lognormal with a comparable parameters: The average pulse interval,

${ }^{2}$ About $15 \%$ of all GRBs are not variable. Clearly the arguments that follow do not apply to this subgroup 
$\bar{\Delta} t=1.3 \mathrm{sec}$ is larger by a factor 1.3 then the average pulse width $\bar{\delta} t=1 \mathrm{sec}$. One also finds that the pulse widths are correlated with the preceding interval $[15,16]$.
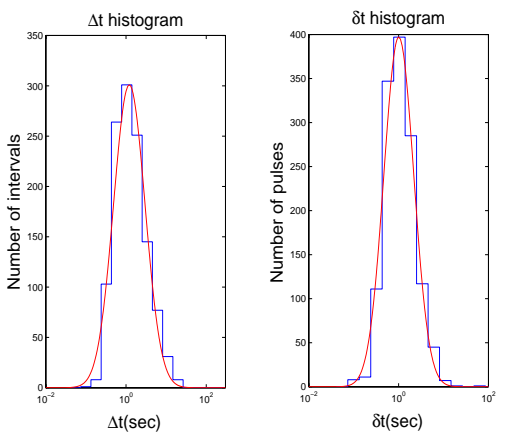

FIGURE 2. The pulse width distribution (right) and the distribution of intervals between pulses (left) (from [16]).

The results described so far are for long bursts. The variability of short $(T<2 \mathrm{sec})$ bursts is more difficult to analyze. The duration of these bursts is closer to the limiting resolution of the detectors. Still most $(\sim 66 \%)$ short bursts are variable with $\delta t / T<0.1$ [17].

\section{TIME SCALES IN GRBS - THEORY}

Consider a spherical relativistic emitting shell with a radius $R$, a width $\Delta$ and a Lorentz factor $\Gamma$. This can be a whole spherical shell or a spherical like section of a jet whose opening angle $\theta$ is larger than $\Gamma^{-1}$. Because of relativistic beaming an observer would observe radiation only from a region of angular size $\Gamma^{-1}$. Photons emitted by matter moving directly towards the observer (point A in Fig. 3) will arrive first. Photons emitted by matter moving at an angle $\Gamma^{-1}$ (point $\mathrm{D}$ in Fig. 3) would arrive after $t_{\text {ang }}=R / 2 c \Gamma^{2}$. This is also the time of arrival of photons emitted by matter moving directly towards the observer but emitted at $2 R$ (point $\mathrm{C}$ in Fig. 3 ). Thus, $t_{\text {rad }} \approx t_{\text {ang }}[13,20]$.

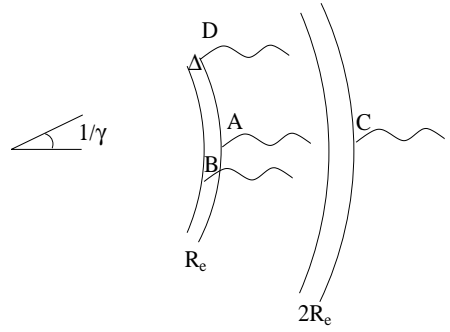

FIGURE 3. Different time scale from a relativistic expanding shell in terms of the arrival times $\left(t_{i}\right)$ of various photons: $t_{\text {ang }}=t_{D}-t_{A}, t_{\text {rad }}=t_{C}-t_{A}$ and $T_{\Delta}=t_{B}-t_{A}$.

At a given point particles are continuously accelerated and emit radiation as long as the shell with a width $\Delta$ is crossing this point. The photons emitted at the front of this shell will reach the observer a time $T_{\Delta}=\Delta / c$ before those emitted from the rear (point 
B in Fig. 3). In fact photons are emitted slightly longer as it takes some time for the accelerated photons to cool. For most reasonable parameters the cooling time is much shorter from the other time scales [21] and I ignore it hereafter.

Light curves are divided into two classes according to the ratio between $T_{\Delta}$ and $t_{\text {ang }} \approx t_{\text {rad }}$. The emission from different angular points smoothes the signal on a time scale $t_{\text {ang. }}$. If $T_{\Delta} \leq t_{\text {ang }} \approx t_{\text {rad }}$ the resulting burst will be smoothed with a width $t_{\text {ang }} \approx t_{\text {rad }}$. Sari and Piran [13] have shown that for external shocks $\Delta / c \leq R / c \Gamma^{2} \approx t_{\text {rad }} \approx t_{\text {ang }}$. External shocks can produce only smooth bursts!

A necessary condition for the production of a variable light curve is that $T_{\Delta}=\Delta / c>$ $t_{\text {ang. }}$ This can be easily satisfied within internal shocks (see Fig 4). Consider an "inner engine" emitting a relativistic wind active over a time $T_{\Delta}=\Delta / c$ ( $\Delta$ is the overall width of the flow in the observer frame). The source is variable on a scale $L / c$. The internal shocks will take place at $R_{s} \approx L \Gamma^{2}$. At this place the angular time and the radial time satisfy: $t_{\text {ang }} \approx t_{\text {rad }} \approx L / c$. Internal shocks continue as long as the source is active, thus the overall observed duration $T=T_{\Delta}$ reflects the time that the "inner engine" is active. Note that now $t_{\text {ang }} \approx L / c<T_{\Delta}$ is trivially satisfied. The observed variability time scale in the light curve, $\delta t$, reflects the variability of the source $L / c$. While the overall duration of the burst reflects the overall duration of the activity of the "inner engine".

Numerical simulations [14] have shown that not only the time scales are preserved but the source's temporal behavior is reproduced on an almost one to one basis in the observed light curve. I return to this point in the next section where I describe a simple toy model that explains this result.

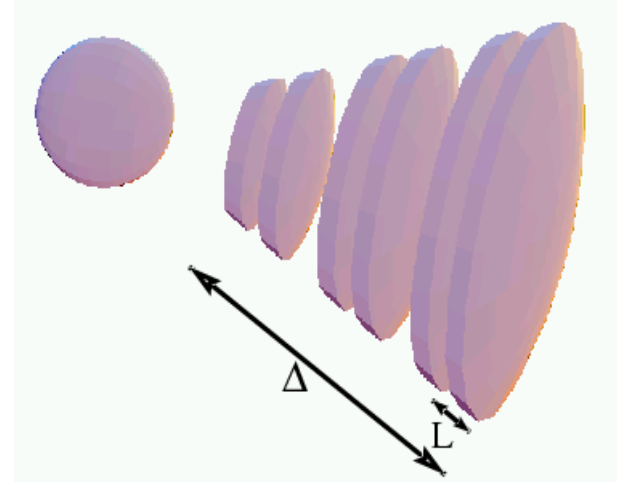

FIGURE 4. The internal shocks model (from [22]). Faster shells collide with slower ones and produce the observed $\gamma$ rays. The variability time scale is $L / c$ while the total duration of the burst is $\Delta / c$.

\section{AN INTERNAL SHOCKS TOY MODEL}

The discovery $[15,16]$ that the distribution of pulse widths and pulse separations are comparable and that there is a correlation between the pulse width and the preceding interval provides an independent evidence in favor of the internal shocks model. Fur- 
thermore it suggests that the different shells emitted by the internal engine are most likely "equal energy" rather than "equal mass" shells.

Both features arise naturally within the internal shocks model [18] in which both the pulse duration and the separation between the pulses are determined by the same parameter. We outline here the main arguments showing that. Consider two shells with a separation $L$. The slower outer shell Lorentz factor is $\Gamma_{1}=\Gamma$ and the inner faster shell Lorentz factor is $\Gamma_{2}=a \Gamma$ ( $a>2$ for an efficient collision), both in the observer frame. The shells' are ejected at $t_{1}$ and $t_{2} \approx t_{1}+L / c$. The collision takes place at a radius $R_{S} \approx 2 \Gamma^{2} L$ (Note that $R_{S}$ does not depend on $\Gamma_{2}$ ). The emitted photons from the collision will reach the observer at time (omitting the photons flight time, and assuming transparent shells):

$$
t_{o} \approx t_{1}+R_{S} /\left(2 c \Gamma^{2}\right) \approx t_{1}+L \approx t_{2} .
$$

The photons from this pulse are observed almost simultaneously with a (hypothetical) photon that was emitted from the "inner engine" together with the second shell (at $\left.t_{2}\right)$. This explains why Kobayashi et al [14] find numerically that for internal shocks the observed light curve replicates the temporal activity of the source.

Consider now four shells emitted at times $t_{i}(i=1,2,3,4)$ with a separation of the order of $L$ between them. Assume that there are two collisions: between the first and the second shells and between the third and the fourth shells. The first collision will be observed at $t_{2}$ while the second one will be observed at $t_{4}$. Therefore, $\Delta t \approx t_{4}-t_{2} \approx 2 L / c$. Now assume a different collision scenario, the second and the first shells collide, and afterward the third shell takes over and collide with them (the forth shell does not play any roll in this case). The first collision will be observed at $t_{2}$ while the second one will be observed at $t_{3}$. Therefore, $\Delta t \approx t_{3}-t_{2} \approx L / c$. Numerical simulations [18] show that more then $80 \%$ of the efficient collisions follows one of the two scenarios described above. Therefore one can conclude that:

$$
\Delta t \approx L / c
$$

Note that this result is independent of the shells' masses.

The pulse width is determined by the angular time (ignoring the cooling time): $\delta t=R_{s} /\left(2 c \Gamma_{s}^{2}\right)$ where $\Gamma_{s}$ is the Lorentz factor of the shocked emitting region. If the shells have an equal mass $\left(m_{1}=m_{2}\right)$ then $\Gamma_{s}=\sqrt{a} \Gamma$ while if they have equal energy $\left(m_{1}=a m_{2}\right)$ then $\Gamma_{s} \approx \Gamma$. Therefore:

$$
\delta t \approx\left\{\begin{aligned}
R_{S} / 2 a \Gamma^{2} c \approx L / a c & \text { equal mass } \\
R_{S} / 2 \Gamma^{2} c \approx L / c & \text { equal energy. }
\end{aligned}\right.
$$

The ratio of the Lorentz factors $a$, determines the collision's efficiency. For efficient collision the variations in the shells Lorentz factor (and therefore $a$ ) must be large.

It follows from Eqs. 2 and 3 that for equal energy shells the $\Delta t-\delta t$ similarity and correlation arises naturally from the reflection of the shells initial separation in both variables. However, for equal mass shells $\delta t$ is shorter by a factor of $a$ than $\Delta t$. Since $a$ has a large variance this would wipes off the $\Delta t-\delta t$ correlation. This suggests that equal energy shells are more likely to produce the observed light curves. 


\section{IMPLICATIONS OF THE INTERNAL SHOCKS MODEL TO THE INNER ENGINE}

The results presented above show that within the internal shocks model the GRB light curve follows the activity of the internal engine. This, in turn, implies that the internal engine must have a rather short typical time scale (as implied by the variability time scale) of about $1 \mathrm{msec}$ or less and a long over all activity time scale (as implied by the duration of the bursts) of several dozen seconds or even a thousand seconds in some cases. The first time scale suggest that GRBs involve a compact object, most likely a black hole. The second time scale indicates activity much longer than the typical dynamical time scale of a compact object and suggest that the "inner engine" involves accretion onto a black hole, with the duration of the burst being the accretion time.

\section{IMPLICATIONS FROM ACCRETION THEORY}

Several scenarios could lead to a black hole - massive accretion disk system. This could include mergers (NS-NS binaries [12, 23], NS-BH binaries [24] WD-BH binaries [25], BH-He-star binaries [26]) and models based on "failed supernovae or "Collapsars" $[9,10,11]$. Narayan, Piran and Kumar [27] (hereafter NPK01) have shown that accretion theory suggests that from all the above scenarios only Collapsars could produce long bursts and only NS-NS (or NS-BH) mergers could produce short bursts. I outline here the essence of their argument.

NPK01 considered a generic accretion model of a GRB in which a mass, $m_{d \odot}$, goes into orbit around a relativistic star of mass $3 m_{\odot}$. The orbiting mass is initially inserted into a torus at a radius $r_{\text {out }} R_{S}$, where $R_{S}$ is the Schwarzschild radius of the central star: $R_{S}=2 G M / c^{2}=8.85 \times 10^{5} m_{3} \mathrm{~cm}$. Starting from the initial toroidal configuration, the mass spreads out by viscosity and becomes an accretion flow extending from $r=1$ (the horizon of the central black hole) to $r \sim r_{\text {out }}$.

If the accretion disk is larger than a few tens or hundreds of Schwarzschild radii, the accretion will proceed via a convection-dominated accretion flow (CDAF) in which most of the matter escapes to infinity rather than falling onto the black hole (see e.g. [28]) this poses a problem for models in which "large" $(r>100)$ disks arise. Ball, Narayan and Quataert [29] estimate the mass accretion rate in the CDAF is estimated to be $\dot{m}$

$$
\dot{m}=3.39 \times 10^{4} m_{3}^{-1} m_{d} r_{\text {out }}^{-5 / 2} .
$$

The accretion time scale is not simply equal to $m_{d} / \dot{m}$. The reason is that much of the mass in a CDAF actually flows out of the system rather than into the central black hole. To estimate the accretion time, $t_{a c c}$, [27] use the turbulent velocity of the connective blobs. They find:

$$
t_{\text {acc }} \approx=4.17 \times 10^{-4} \alpha_{a}^{-1} m_{3} r_{\text {out }}^{3 / 2} \mathrm{~s},
$$

where $\alpha_{a}$ is the dimensional parameter of the accretion viscosity. This scaling estimates agrees with numerical simulations of CDAFs [30]. One can notice immediately that long accretion times require large disks and hence if the duration of the burst is determined 
by the duration of the accretion the accretion torus must be large. However, in this case the mass, $m_{a c c}=t_{a c c} \dot{m}$, accreted by the black hole, are very small:

$$
\begin{aligned}
m_{a c c} & =m_{d}, & r_{\text {out }} \leq 14.1 \alpha_{a}^{-1} m_{d}, \\
& =14.1 \alpha_{a}^{-1} m_{d} r_{\text {out }}^{-1}, & r_{\text {out }}>14.1 \alpha_{a}^{-1} m_{d} .
\end{aligned}
$$

When $r_{\text {out }}$ is large, the accreted mass is much less than $m_{d}$. The reason is that the bulk of the mass is ejected from the system, flowing out at $r \sim r_{\text {out }}$. The energy for the ejection is provided by convective energy flux from the interior of the flow. [27] find that CDAF is possible only if:

$$
r_{\text {out }}>118 \alpha_{a}^{-2 / 7} m_{3}^{-1} m_{d}^{3 / 7}
$$

If the mass in the accretion flow is initially at a radius greater than the above limit, then the flow will become a CDAF at $r=r_{\text {out }}$, and for all $r<r_{\text {out }}$.

Within the inner region of the disk, very near the black hole the temperatures a high enough so that the disk can cool by emitting thermal neutrinos [31]. This neutrinodominated accretion flow (NDAF) is efficient. An NDAF behaves like a thin accretion disk and use the basic theory of thin disks to estimate the accretion time and the accretion rate $[31,27]$ :

$$
\begin{aligned}
t_{a c c} & =2.76 \times 10^{-2} \alpha_{a}^{-6 / 5} m_{3}^{6 / 5} r_{\text {out }}^{4 / 5} \mathrm{~s}, \\
\dot{m} & =36.2 \alpha_{a}^{6 / 5} m_{3}^{-6 / 5} m_{d} r_{\text {out }}^{-4 / 5}
\end{aligned}
$$

In a cooling-dominated thin disk, very little mass is expected to be lost to outflows, so nearly all the mass in the disk to be accreted by the star, i.e.

$$
m_{a c c} \approx m_{d}
$$

These results assume that the disk is gas pressure dominated. Slightly different resutls are obtained if degeneracy pressure takes over the gas pressure [27]. DiMatteo, Narayan and Perna [32] considered with the details of the neutrino transport within the inner parts of the disk. They find that the disk can become optically thick to neutrinos in its most inner parts. If the neutrinos are sufficiently trapped then energy advection becomes once more important.

NS-NS and BH-NS merger models, with $\left(r_{\text {out }}, m_{d}\right)=(10,0.1)$ and $(10,0.5)$ (see [31]), are well inside the NDAF zone and, according to our calculations, are capable of producing GRBs. However, this is only if the black hole is small (few $M_{\odot}$ ). If the black hole is larger than $\sim 10 M_{\odot}$ its Schwarszchild radius becomes too large and there is not enough "room" for an NDAF solution around it. Moreover, the neutron star in this case is swallowed whole by the $\mathrm{BH}$ and it is not tidally disrupted to create an accretion disk. On the other hand, unless the viscosity is very small, such disks cannot produce long bursts lasting hundreds or even tens of seconds. This suggests that NS-NS mergers and BH-NS mergers with smaller BH masses produce short GRBs but not long ones.

Other merger models, specifically the BH-WD and the BH-He star merger models, would appear not to be viable GRB engines. As the secondaries in these systems are not compact, they would form accretion flows with large values of $r_{\text {out }}$. For instance, [31] 
estimate $r_{\text {out }} \sim 3000$ for a BH-WD binary and $r_{\text {out }} \sim 5000$ for a BH-He star binary. At these radii, the accretion flow will be a very extended CDAF and hardly any mass will be accreted. Although the time scales of these models are consistent with long bursts, the extremely small value of $m_{a c c}$ suggests that these models do not produce GRBs of any kind.

In binary mergers, one can expect that a certain fixed amount of mass is instantaneously input into the accretion flow. The Collapsar model [11] corresponds to a different scenario in which mass is steadily fed over a period of time by fallback from the supernova explosion. [11] show that the time scale of the GRB is set by the physics of fallback rather than by accretion. Further, the time scales they obtain are consistent with observations of long GRBs. While the time scale may be set by fallback, the efficiency of the burst still depends on the nature of the post-fallback accretion. Efficient accretion, where most of the fallback material reaches the black hole, is possible only if $r_{\text {out }}$ is small and falls within the NDAF zone. If Collapsars have a distribution of $r_{\text {out }}$ and $m_{d}$, then only those systems that have $r_{\text {out }} \leq 100 \alpha^{-2 / 7} m_{3}^{-1}$ will make bursts. Systems with larger angular momentum, and hence larger $r_{\text {out }}$, will form CDAFs and will eject most of the mass.

\section{CONCLUSIONS}

I cannot provide a recipe for a GRB "inner engine". However I can list the specifications of this engine (for a long variable GRB). It must satisfy the following conditions:

- It should accelerate $\sim 10^{51} \mathrm{ergs}$ to a variable relativistic flow with $\Gamma>100$.

- It should collimate this flow, with a varying degree of collimation (up to $1^{o}$ ).

- It should be active from several seconds up to several hundred seconds (according to the duration of the observed burst).

- It should vary on a time scale of a fraction of a second (corresponding to the duration of a typical pulse within the burst).

- Different shells of matter should have a comparable energy and their different Lorentz factors should arise due to a modulation of the accelerated mass.

- At times the engine should stop for several dozen seconds (resulting in a quiescent periods).

The natural model that satisfies these conditions is an accretion disk around a new born black hole. Arguments based on accretion theory [27] suggest that:

- Long bursts are produced by a Collapsar in which the duration of the activity of the disk depends on the time that matter fallback on the disk from the supernova explosion.

- Short bursts are produced by Neutron star binary mergers (or black hole-neutron star mergers) an in those the duration of the bursts is dictated by the duration of the accretion of the disk onto the black hole. 


\section{ACKNOWLEDGMENTS}

This research was supported by a grant from the US-Israel Binational Science Foundation.

\section{REFERENCES}

1. T. Piran, Physics Reports, 314, 575 (1999).

2. T. Piran, Rev. Mod. Phys, 76, 1143 (2004)..

3. T. Galama et al., Nature, 395, 70, (1998).

4. Bloom, J. S. et al., Nature, 401, 453 (1999).

5. Reichart, D. E. 1999, ApJ, 521, L111, (1999).

6. Galama, T. J. et al., ApJ, 536, 185 (2000).

7. K. Z. Stanek, T. Matheson, P. M. Garnavich et al., Ap. J. Lett., , 591, L17-L20 (2003).

8. J. Hjorth, J. Sollerman, P. Møller et al., Nature, , 423, 847-850 (2003).

9. S. E. Woosley, Ap. J., 405, 273 (1993).

10. B. Paczynski, Ap. J. Lett., 494, L45 (1998).

11. A. I. MacFadyen and S. E. Woosley, Ap. J., 524, 262 (1999).

12. D. Eichler, M. Livio, T. Piran, and D. N. Schramm, Nature, 340, 126 (1989).

13. R. Sari and T. Piran, Ap. J., 485, 270 (1997).

14. S. Kobayashi, T. Piran, and R. Sari, Ap. J., 490, 92 (1997).

15. E. Nakar and T. Piran, astro-ph/0103011, GRBs in the Afterglow Era, Eds. E. Costa, F. Fronteira and K. Hjorth, (Springer) (2001).

16. E. Nakar, and T. Piran, Mon. Not. RAS, , 331, 40-44 (2002).

17. E. Nakar, and T. Piran, Mon. Not. RAS, , 330, 920-926 (2002).

18. E. Nakar, and T. Piran, Ap. J. Lett., , 572, L139-L142 (2002).

19. E. Ramirez-Ruiz, and A Melroni, MNRAS, 320, K25 (2001).

20. E. E. Fenimore, C. D. Madras, and S. Nayakshin, Ap. J., 473, 998, (1996).

21. R. Sari, R. Narayan and T. Piran, Ap. J., 473, 204, (1996).

22. R. Sari, PhD thesis, (1998)

23. R. Narayan, B. Paczynski, and T. Piran, Ap. J. Lett., , 395, L83-L86 (1992).

24. B. Paczynski, Acta Astronomica, 41, 257-267 (1991).

25. C. L. Fryer, S. E. Woosley, M. Herant, and M. B. Davies, Ap. J., , 520, 650-660 (1999).

26. C. L. Fryer, and S. E. Woosley, Ap. J. Lett., , 502, L9-+ (1998).

27. R. Narayan, T. Piran and P. Kumar, Ap. J., 557, 949, (2001).

28. J. M. Stone, J. E. Pringle, and M. C. Begelman, Mon. Not. RAS, , 310, 1002-1016 (1999).

29. Ball, G. H., Narayan, R., \& Quataert, E. Ap. J., , 552, 221, (2001).

30. I. V. Igumenshchev, and M. A. Abramowicz, Ap. J. Supp., , 130, 463-484 (2000).

31. R. Popham, S. E. Woosley, and C. Fryer, Ap. J., , 518, 356-374 (1999).

32. Di Matteo, T., Perna, R., \& Narayan, R. Ap. J., , 579, 706 (2002). 